\title{
Holography as a tool for advanced learning of optics and photonics
}

\section{Victor Dyomin, Igor Polovtsev, Alexey Olshukov}

Victor V. Dyomin, Igor G. Polovtsev, Alexey S. Olshukov, "Holography as a tool for advanced learning of optics and photonics," Proc. SPIE 9666, 11th Education and Training in Optics and Photonics Conference, 96660M (5 June 2009); doi: 10.1117/12.2207969

SPIE Event: Eleventh International Topical Meeting on Education and Training in Optics and Photonics, 2009, St. Asaph, United Kingdom 


\title{
Holography as a tool for advanced learning of optics and photonics
}

\author{
Victor V. Dyomin, Igor G. Polovtsev, Alexey S. Olshukov \\ Tomsk State University \\ 36 Lenin Avenue, Tomsk, 634050, Russia \\ Tel/fax: 73822 412573, dyomin@tsu.ru
}

\begin{abstract}
Laboratory works on holograms recording, reconstruction and interpretation are useful for two reasons. Firstly, holography is widely used in science and engineering. Secondly, training labs in holography require complex applying of knowledge on interference, diffraction, coherency and other domains of optics.

Educational kit and methodological instructions for optical experiments were presented in the previous paper ${ }^{1}$. The desktop holographic camera described in this paper is one of the additional functional units of the kit. The desktop holographic camera does not require additional protection against vibrations even if the exposure time is several minutes. This is a compact holographic installation for recording Denisyuk holograms.

Two experiments are described in the paper to illustrate the usefulness of holographic laboratory works. The first one is a recording and reconstruction of a Denisyuk hologram. The second one is a recording and interpretation of a double-exposure interferogram when the holoplate is sagged due to loading between exposures.

Also included in the paper are holographic setup and laboratory works on digital holography. These experiments require, in addition, complex applying of knowledge on photo receivers, CCD and other domains of photonics.
\end{abstract}

Keywords: educational kit, training labs, holography, desktop holographic camera, Denisyuk hologram, double-exposure interferogram, digital holography.

\section{INTRODUCTION}

The educational kit "UMOG" and its methodological instructions have been presented in previous papers ${ }^{1,2}$. Methodological instructions provided with this set include the list of demonstrations and laboratory works as well as links between various experiments and phenomena. Methodological instructions give recommendations for more than 50 demonstrations and practical works in various domains: light diffraction, interference of light, holography, geometric optics, Fourier optics, polarization effects, optics of spectra, fiber optics, as well as a combination of the effects.

A picture of the set is shown in fig.1.

The desktop holographic camera (fig. 2) is one of the additional functional units of the kit.

Laboratory works on holograms recording, reconstruction and interpretation are useful for two reasons. Firstly, holography is widely used in science and engineering. Secondly, training labs in holography require complex applying of knowledge on interference, diffraction, coherency and other domains of optics.

Therefore this paper is devoted to holographic training labs. This paper is based on more than 15 years' experience of carrying out training labs in holography at the university. 


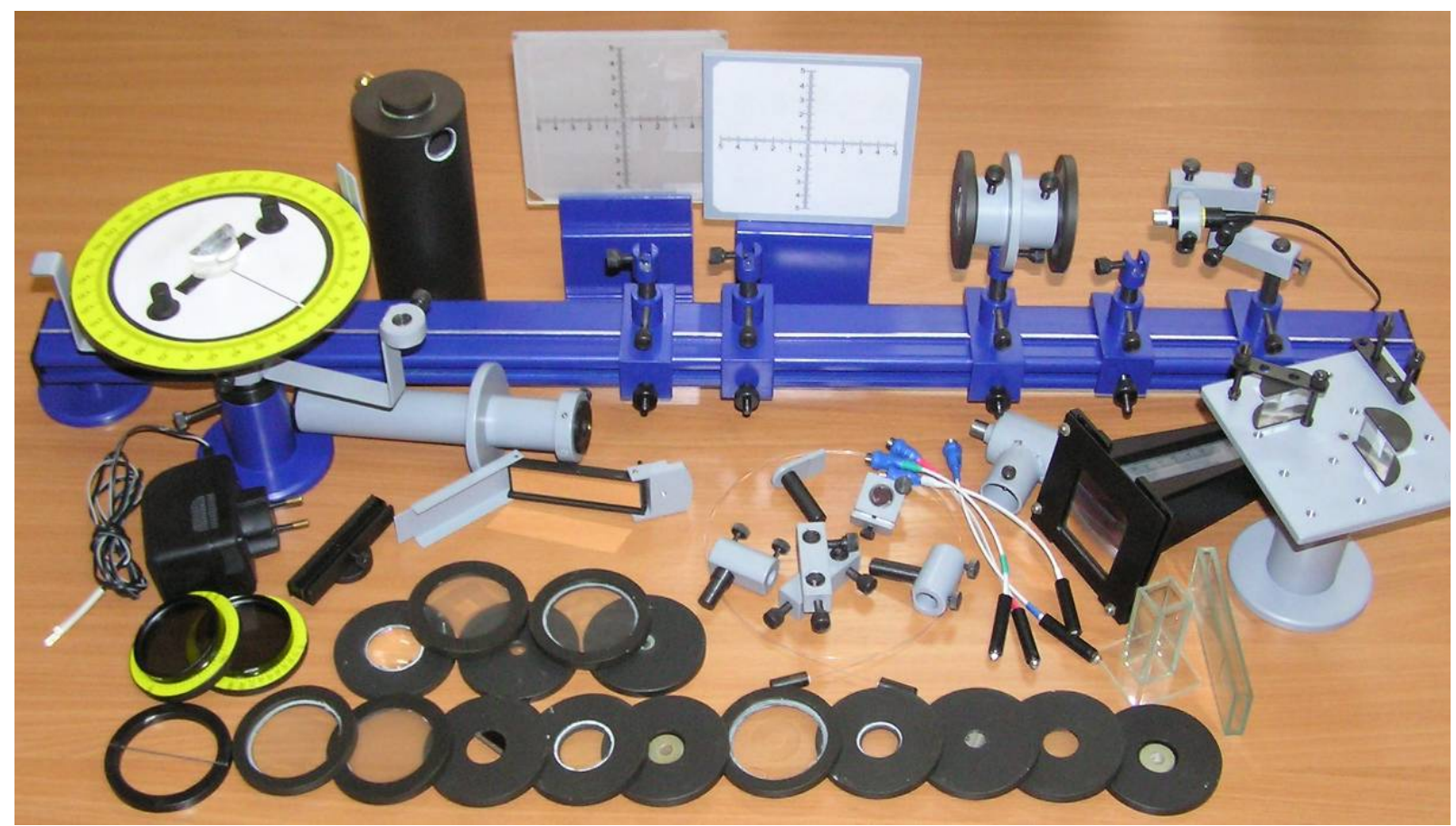

Fig. 1. The base set of equipment

\section{DESKTOP HOLOGRAPHIC CAMERA}

Normally, holograms are recorded using huge installations that should provide protection against vibrations if a cw laser is used as a light source. This is necessary when recording holograms of large scenes or moving objects. At the same time there are many applications where no such cumbersome instrumentation is needed. Among such applications, manufacturing of holographic filters and diffraction gratings can be mentioned. Creation of archives of small objects and production of souvenirs are also examples of such an application of holography. In these cases it is necessary, as a rule, to make small holograms of a stationary object.

We have developed a desktop holographic camera for training labs on holography. But it can be used for above mentioned application as well. This is a compact holographic installation for recording Denisyuk holograms (fig.2). The size of this instrument is 300 by 250 by $500 \mathrm{~mm}$ and its weight does not exceed $10 \mathrm{~kg}$. Holoplates ranging in size from 76 by 76 to 127 by $127 \mathrm{~mm}$ can be used for holographing. The depth of a scene that can be recorded is about $50 \mathrm{~mm}$. The cw He-Ne laser used in the camera delivers $2 \mathrm{~mW}$ power that is quite sufficient for recording holograms of such a size.

The instrument doesn't require additional protection against vibrations even if the exposure time is several minutes. This is achieved by using a vertical frame for assembling a laser (2) and other optical elements of the instrument as well as by a rigid coupling of the object plane and the plane where the photographic material is placed to record the hologram. Mirrors (3) are designed for alignment of the device. Laser beam is expanded by a microlens (4). A microlens can be replaced by a collimator. The holder of a holoplate (5) can be regulated to use holoplates of various sizes - from 76 by 76 to 127 by $127 \mathrm{~mm}$. An object holder can be placed in a desirable position and then fixed by two screws. An object (6) is placed inside the case (1) to protect the object field against the air flows. To record a Denisyuk hologram the pattern (7) of interference between object and reference wave is recorded in thick holographic emulsion (5).

Two light sources from the kit (UMOG, fig.1) can be used in the desktop holographic camera: a laser semiconductor diode $(\lambda=655 \mathrm{~nm})$ and a He-Ne laser $(\lambda=633 \mathrm{~nm})$. Experimentally it is found that the semiconductor laser coherence allows us to record Denisyuk hologram but the quality of reconstructed holographic image is not high. Therefore we use the He-Ne laser in the majority of our holographic experiments. 


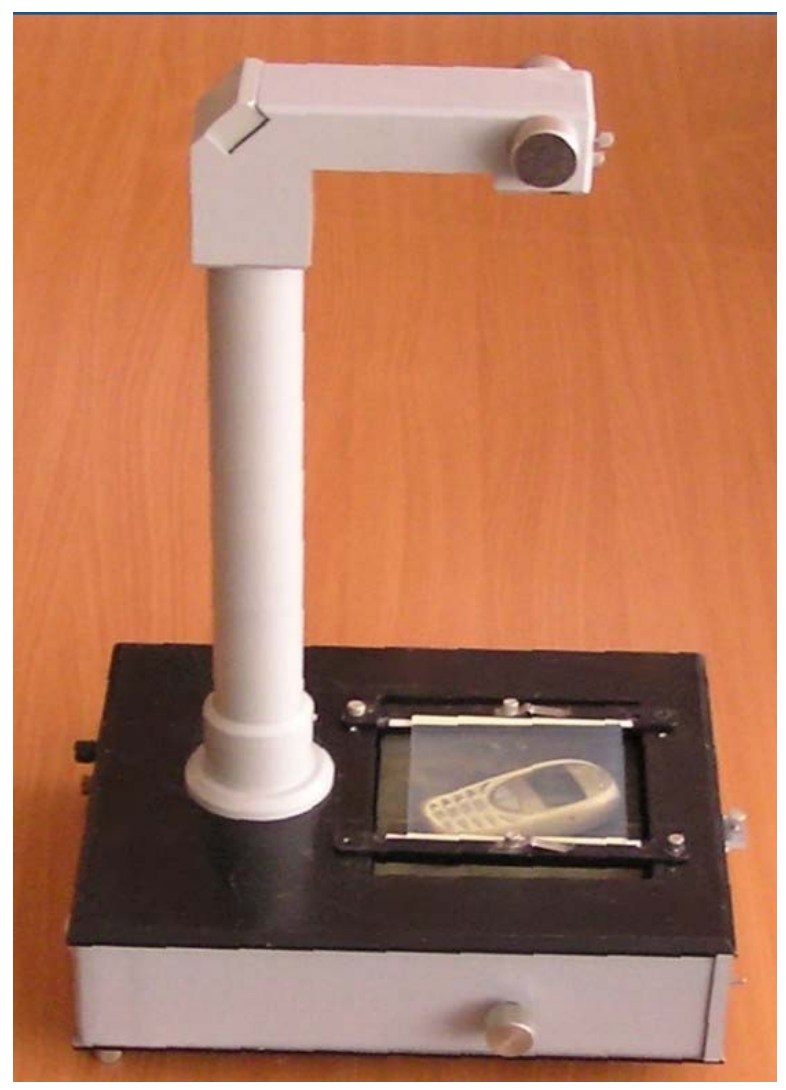

a

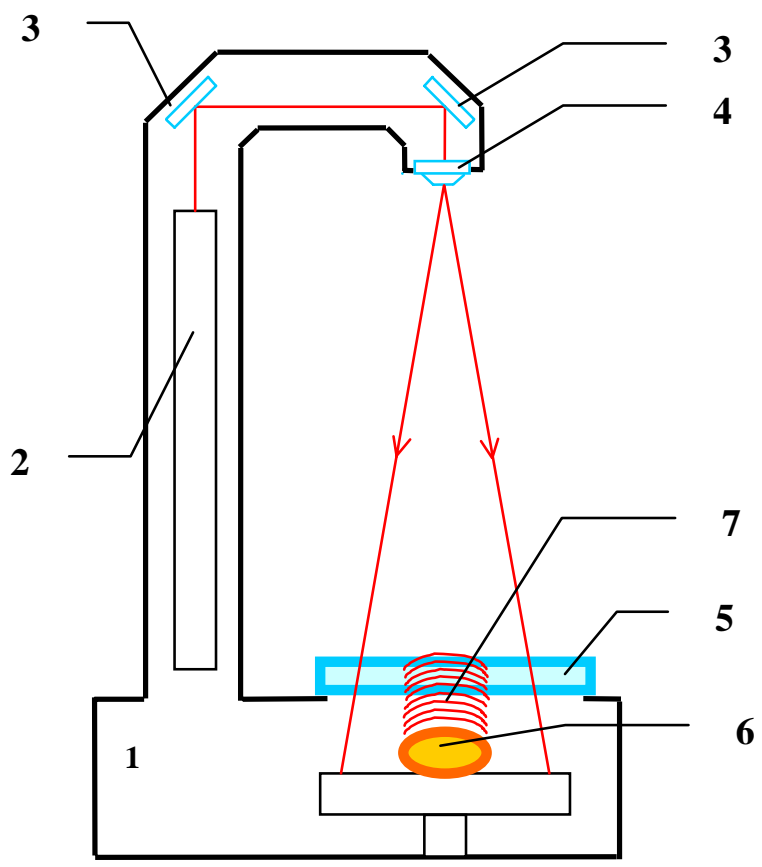

b

Fig.2. Desktop holographic camera.

$a$ - picture of camera; $b$ - functional scheme.

1 - case, 2 - laser, 3 - mirrors, 4 - microlens, 5 - holographic plate, 6 - object of holographing, 7 - pattern of interference between object and reference waves.

\section{RECORDING OF DENISYUK HOLOGRAMS AND HOLOGRAPHIC INTERFEROGRAMS}

In order to simplify the use of the instrument, the holograms are recorded using coaxial optical arrangement (fig $2 \mathrm{~b}$ ). It is known that a "transparent" photoplate has to be used in such scheme because an object beam is passed through the plate, reflected from an object and returned to the plate. To record the holograms we use silver halide plates such as PFG-03 ("Slavich", Russia). The resolution of these photoplates is up to $5000 \mathrm{~mm}^{-1}$. In addition, their thickness is acceptable for recording Denisyuk holograms. We use developer GP-2 for processing the hologram.

The exposure time in our experiments ranged from 30 seconds to 5 minutes. This fact demonstrates that no additional protection against vibration is needed.

We have been using the desktop holocamera for about 15 years in student labs on holography and optical data processing. Hundreds of holograms have been recorded with the above set-up. Various objects were recorded. It was found that holograms of diffusely reflecting objects allow reconstructing most qualitative holographic images in a white light. It is obvious that because of the simple functioning of the instrument, it is impossible to produce holograms of slightly reflecting objects. 
Double-exposed holograms are also recorded in our experiments. This same optical scheme is used. A plane mirror is used as an object (6) (Fig. 2). A small weight is located on the holoplate between two exposures. Therefore two holograms are recorded on the holoplate. One hologram corresponds to the initial stage of the holoplate, another hologram is recorded on the loaded holoplate. Two waves are restored from the hologram so we can observe the interference fringes under reconstruction stage. Various kinds of interference fringes are reconstructed in a white light. Certainly, the shape of the fringes depends on the character of deformation of the holoplate between the exposures. Such an agreement between the appearance of the fringes and the position of the weight is observed in our experiments.
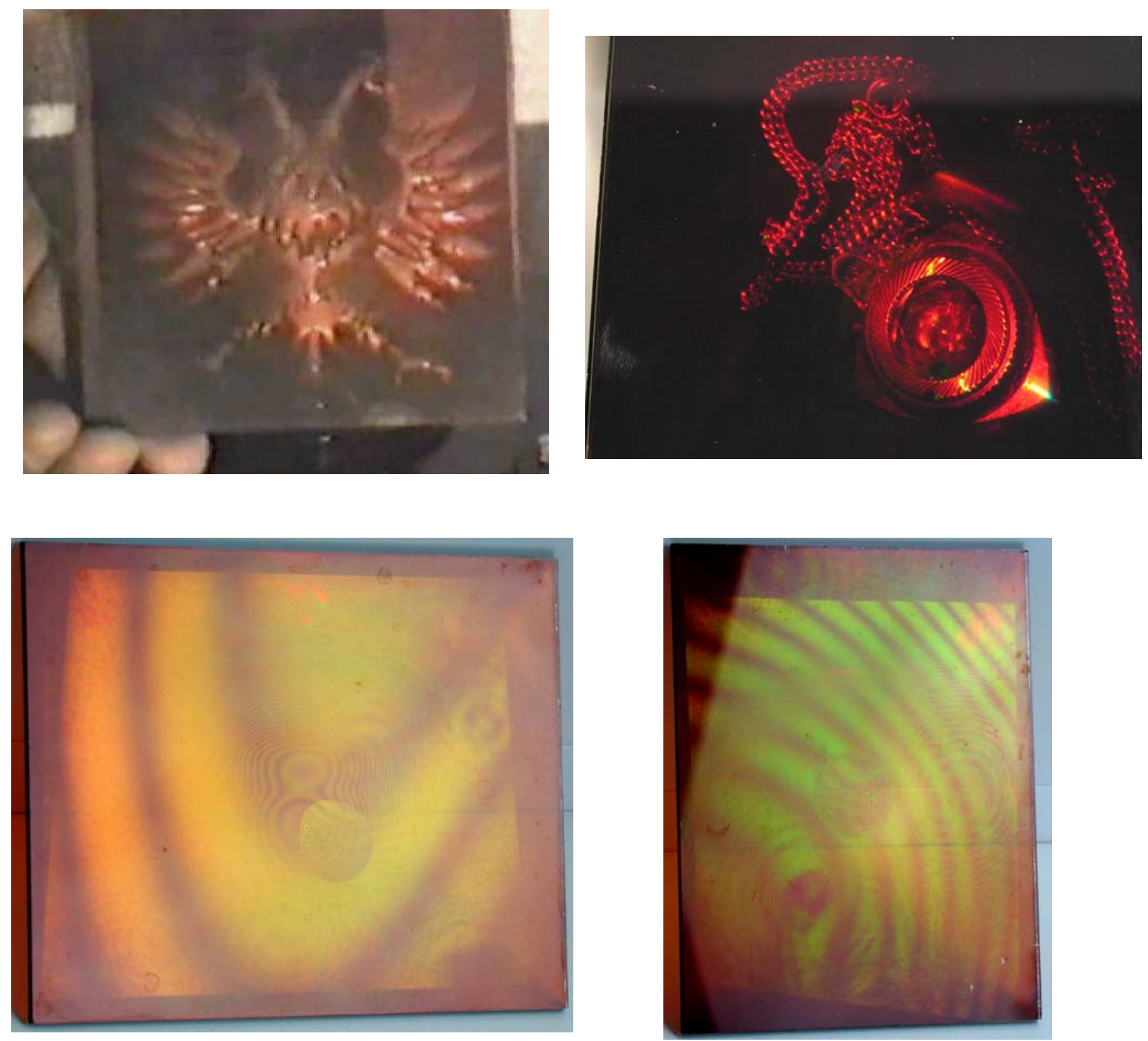

Fig. 3. Examples of holograms and interferograms 
A plane mirror could also be used as an object when a reflective holographic filter is recording.

In addition to holographing according to Denisyuk scheme the instrument enables the recording of holographic gratings and holograms of some simple objects using Leit and Upatnieks scheme as well. In this case an additional holder should be used. The beam splitting is carried out by the prism. In order to test the device we have recorded a number of holographic gratings, both absorption and surface-relief ones. We used Kodak D-19 for developing the photoplate and Kodak R-10 to make bleached holographic gratings. The spatial frequency of the gratings did not exceed $1000 \mathrm{~mm}^{-1}$ in our experiments. The diffraction efficiency of these gratings and the diffraction efficiency of the gratings recorded by the typical vibration-resistant set-up have the same order of magnitude.

In our training labs students independently carry out the whole holographic procedure. They adjust the scheme, expose and develop holoplate, reconstruct and explore both real and virtual images of object. When students make the double-exposed hologram they should determine the type of deformation of holoplate between exposures and measure its central deflection (sag) using interference pattern in the reconstructed image.

\section{DIGITAL HOLOGRAPHY EXPERIMENTS}

Digital holography (when the hologram records on CCD and the holographic image reconstruction is done on the computer) provides additional important opportunities of holography. The following advantages can be mentioned for this technique:

- A possibility to create holographic video;

- $\quad$ A possibility to transmit digital holograms by communication lines;

- A possibility to calculate the phase of reconstructed wave.

At present, digital holography is quite a traditional method, therefore we suggest to represent it in student training labs.

Optical scheme for digital hologram recording is presented in fig. 4. In-line holographic scheme is used because of the limited resolution of CCD-camera. For simplicity we use the scheme with direct illumination of object.

Laser beam expanded to necessary diameter illuminates the object scene (3). Laser radiation scattered by objects is the object wave. The other part of radiation passed by the objects is the reference wave. The pattern of interference of these coherent waves is recorded by CCD - camera (4) and stored in the computer (5) memory.

Of course, technical parameters of CCD-camera must provide the correct registration of the interference pattern. For example, in order to register an in-line hologram of a spherical particle with $50 \mu \mathrm{m}$ size, located on the $500 \mathrm{~mm}$ distance, the CCD-camera must meet the following requirements (if the wavelength of laser radiation is $0.63 \mu \mathrm{m}$ ):

- $\quad$ CCD size - lager than $12.6 \mathrm{~mm}$;

- $\quad$ Pixel size - smaller than $6 \mu \mathrm{m}$;

- $\quad$ Bit capacity for pixel - not less than 12.

For training purposes we use quite a cheap camera SK-2005 which is easy in operation.

If the CCD-camera allows the correct registering of the interference pattern of the object and reference waves, we can consider a so-called ideal recording of hologram. The recorded hologram is a 2-D discrete array of intensity values. In the case of an ideal hologram recording this same array can be used as complex amplitude $u\left(x_{1}, y_{1}\right)$ of restored wave just behind the hologram, on reconstruction stage. Therefore the use of diffraction integral (1) allows us to calculate complex amplitude $u\left(x_{2}, y_{2}\right)$ and intensity of restored wave in any plane $\left(x_{2}, y_{2}\right)$ located on a distance $z$ behind the hologram.

$$
u\left(x_{2}, y_{2}\right)=\frac{e^{i k z}}{i \lambda z} \int_{-\infty-\infty}^{\infty} \int_{0}^{\infty} u\left(x_{1}, y_{1}\right) e^{\frac{i k}{2 z}\left(\left(x_{2}-x_{1}\right)^{2}+\left(y_{2}-y_{1}\right)^{2}\right)} d x_{1} d y_{1}
$$

Here $\lambda$ is the wavelength of laser radiation, $k=2 \pi / \lambda$. 
Changing the distance $z$ of reconstruction in integral (1) with specified interval we can observe reconstructed images in various sections of object volume. To get in-focus image of object we should adjust the distance of reconstruction within corresponding distance interval.

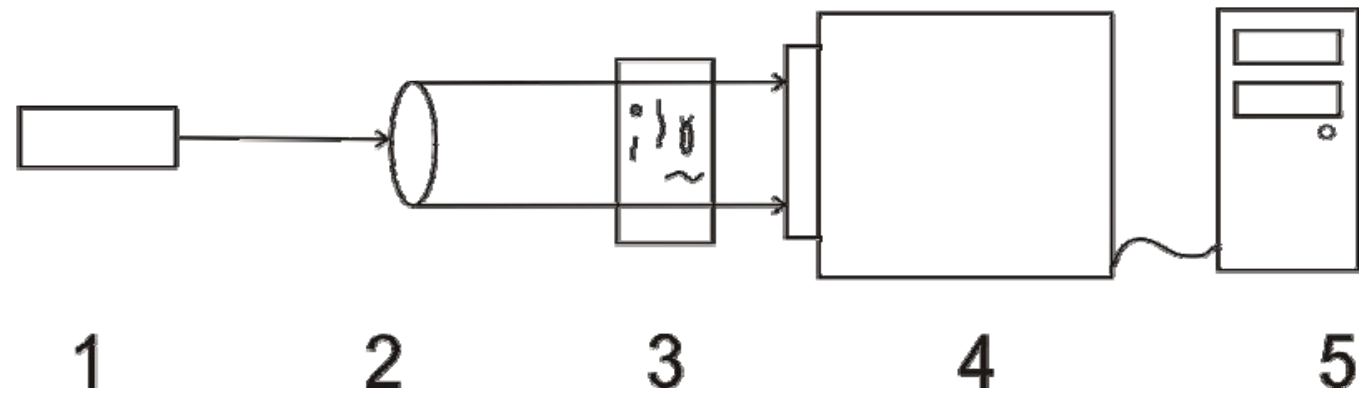

Fig. 4. In-line scheme for digital hologram recording.

1 - laser; 2 - beam expander; 3 - objects; 4 - CCD - camera; 5 - computer.

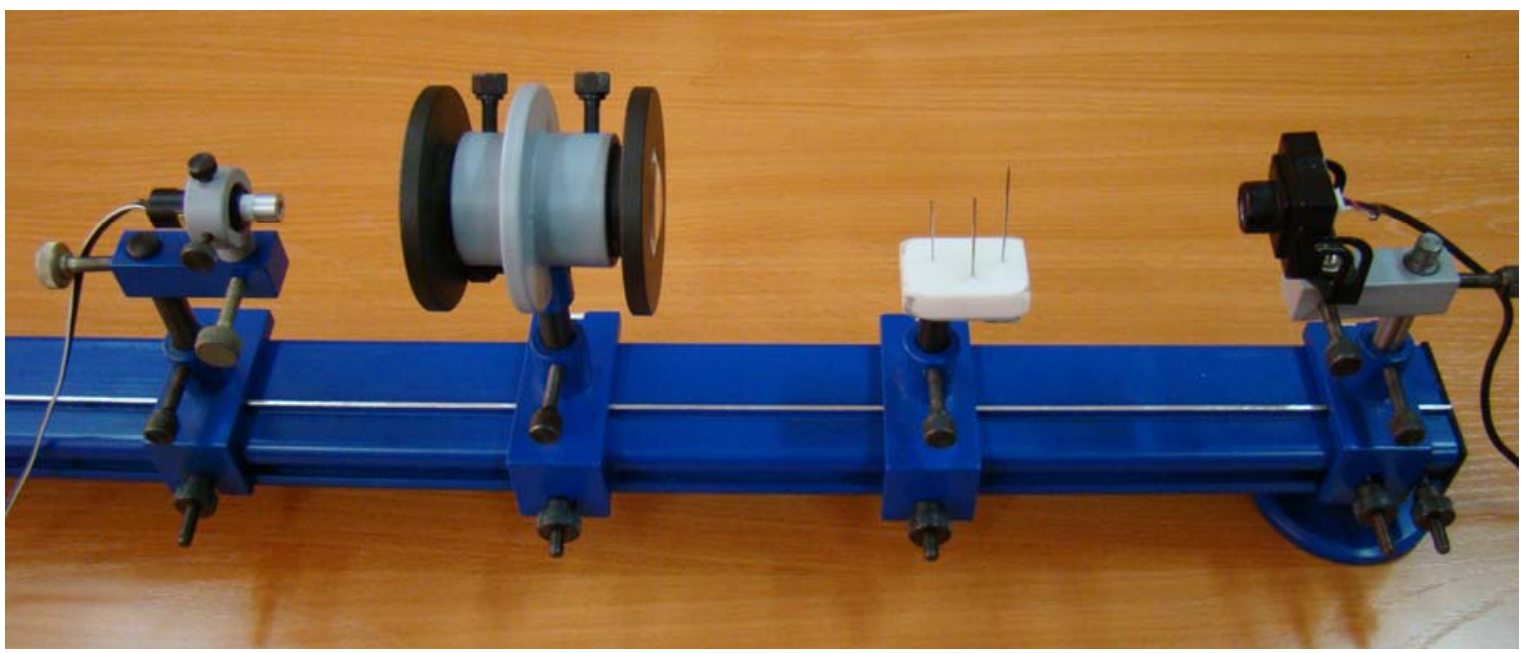

Fig. 5. Set-up for digital hologram recording

The in-line optical scheme described above (fig. 4) is used for holograms recording in student training labs. We use CW He-Ne laser $(\lambda=0.63 \mu \mathrm{m})$ as a source of coherent light. Holograms are recorded by CCD camera SK-2005, with 1/3" CCD matrix, pixel size -9.6 by $7.5 \mu \mathrm{m}$.

The set-up for training lab on digital hologram recording is shown in fig. 5. An optical bench, holders and optical elements from UMOG base set are used for combining the set-up. Three sewing needles located on different distances from the plane of hologram registration are used as objects of holographing.

In this training lab students adjust the scheme and record digital holograms independently. Then they reconstruct the real images of objects using software which calculates the integral (1). To observe a focused image of each needle they change distance of reconstruction in (1). As a result, several images (frames) corresponding to each distance are obtained. Note that if we put these frames together, the video of the refocusing process will be created. This video corresponds to the refocusing process which is observed, for example, when microscope is adjusted to see a sharp image.

Examples of the images reconstructed from digital hologram of three needles are shown in fig. 6 . It is clearly illustrated that the sharp image of each needle is observed on different distances. 


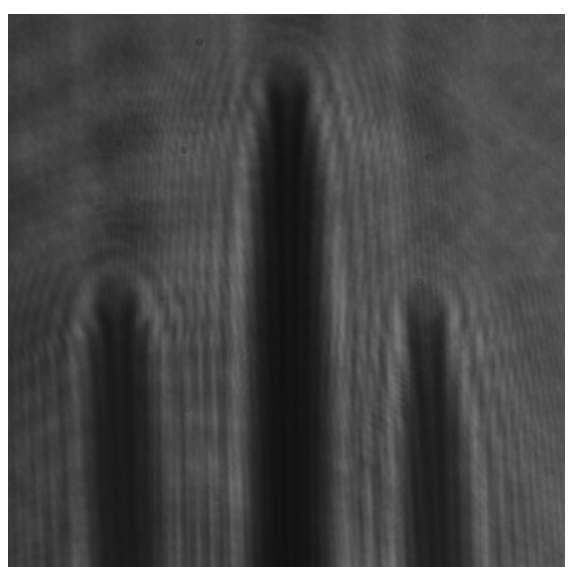

Digital hologram recorded on CCD

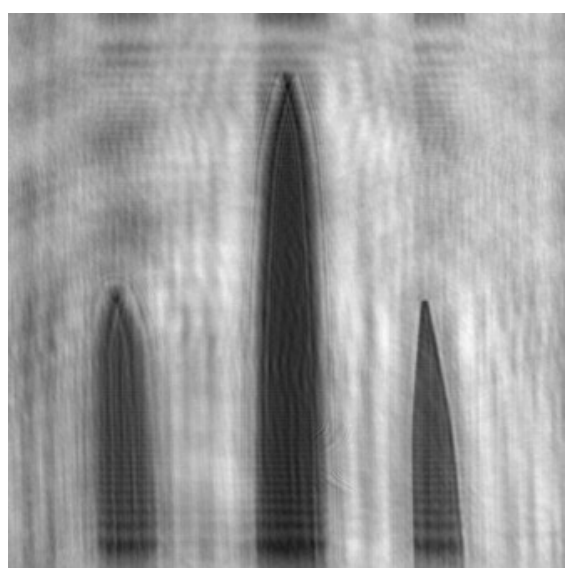

Distance of reconstruction $197 \mathrm{~mm}$, sharp image of right needle is focused

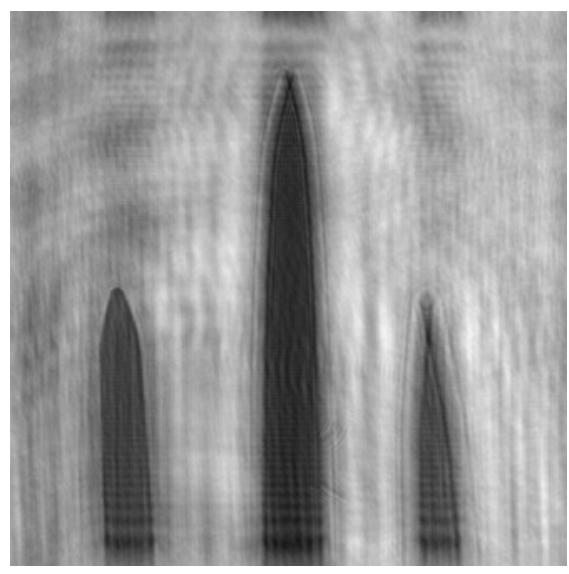

Distance of reconstruction $225 \mathrm{~mm}$, focused image of left needle is observed

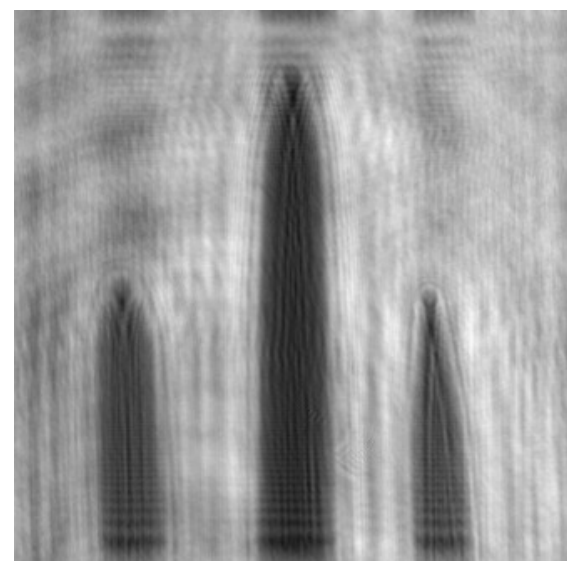

Distance of reconstruction $170 \mathrm{~mm}$, out-of-focus images of needles are observed

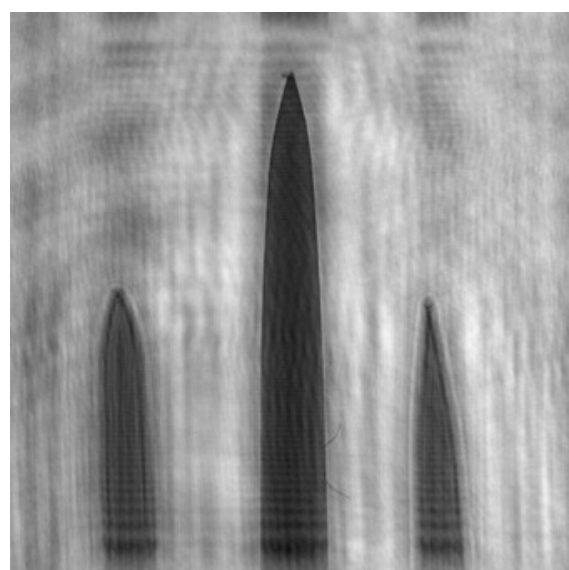

Distance of reconstruction $211 \mathrm{~mm}$, sharp image of middle needle is observed

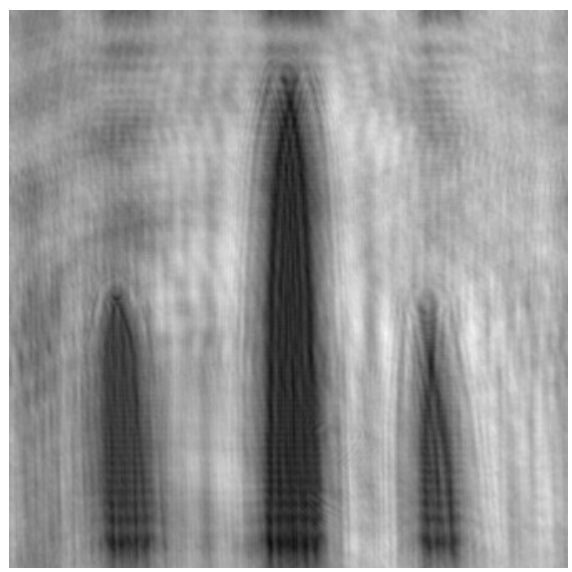

Distance of reconstruction $250 \mathrm{~mm}$, out-of-focus images of needles are observed

Fig. 6. Images reconstructed on various distances. 


\section{CONCLUSION}

This paper outlines several experiments, focusing on recording and reconstruction of Denisyuk hologram as well as digital holograms. The educational kit UMOG ${ }^{1,2}$ is used to prepare these experiments. The methodology of experiments is discussed briefly.

The usefulness of such training labs for the study of optics, photonics, and laser science is confirmed by more than 15 years' experience of conducting these experiments. The holographic training labs promote better understanding of many optical phenomena, such as coherence, interference, diffraction, etc.

\section{REFERENCES}

[1]. Dyomin V.V., Polovtsev I.G. "Educational kit for optical experiments", Tenth International Topical Meeting on Education \& Training in Optics and Photonics, Ottawa, Ontario, Canada, 3-6 June 2007. Chair: Marc Nantel, Ontario Centres of Excellence and OPETA. Proceedings of the 2007 ETOP Conference, (2007).

[2]. Dyomin V.V., Polovtsev I.G. "Set of instrumentation and methodological instructions for practical work in optics", SPIE Proceedings, Vol. 2525, P. 45 - 55 (1995). 